\title{
Current-induced interactions of multiple domain walls in magnetic quantum wires
}

\author{
N. Sedlmayr ${ }^{1 *}$ and V. K. Dugaev ${ }^{2}$, and J. Berakdar ${ }^{1}$ \\ 1 Department of Physics, Martin-Luther-Universität Halle-Wittenberg, \\ Heinrich-Damerow-Str. 4, 06120, Halle, Germany \\ 2 Department of Physics, Rzeszów University of Technology, \\ al. Powstańców Warszawy 6, 35-959 Rzeszów, Poland and \\ Department of Physics and CFIF, Instituto Superior Técnico, \\ TU Lisbon, av. Rovisco Pais, 1049-001, Lisbon, Portugal
}

(Dated: June 4, 2018)

\begin{abstract}
We show that an applied charge current in a magnetic nanowire containing domain walls (DWs) results in an interaction between DWs mediated by spin-dependent interferences of the scattered carriers. The energy and torque associated with this interaction show an oscillatory behaviour as a function of the mutual DWs orientations and separations, thus affecting the DWs' arrangements and shapes. Based on the derived DWs interaction energy and torque we calculate DW dynamics and uncover potential applications of interacting DWs as a tunable nano-mechanical oscillator. We also discuss the effect of impurities on the DW interaction.

PACS numbers: 75.60.Ch,75.75.+a,75.60.Jk
\end{abstract}




\section{INTRODUCTION}

Domain walls (DWs), i.e. regions of noncollinearity separating areas of different homogenous magnetization directions, are important from a fundamental and application point of view $\underline{1} \underline{2}, \underline{3}$. This is particularly the case at low dimensions, as in magnetic nanowires carriers turn out to couple strongly with DWs $\underline{4}$ leading to a marked influence on the wire's transport properties, e.g. DW magnetoresistances in the range of $1000 \%$ were reported $\underline{5,6,7}$. As this coupling is associated with a change of the carriers' spin, it results in a current-induced spin torque acting on the DW and consequently in a current-induced DW motion 1,2 . Based on these facts magnetic nanowires with a series of DWs can be utilized as a "racetrack DW memory" $\underline{\underline{3}}$. The DWs' motion is current-controlled; DWs separated by rather small distances are addressable thus allowing for a high memory density.

In another context it is established that strong carrier scattering and interference results in long-range interactions between impurities on metal surfaces. This interaction governs the impurities geometric arrangements and growth $1,9,10,11,12,13$. The question of whether and how the carriers' spin dependent scattering mediates interactions between DWs is still outstanding and should be addressed here. Clearly, the answer is of vital importance for a high-density nanowire-based racetrack memory and adds a new twist on interferencemediated interactions. We focus on the current-induced part of the coupling between neighboring DWs in a magnetic nanowire. Based on our results we identify the following mechanism of the DWs coupling: Upon scattering from the first DW a carrier spiral spin density builds up. This acts as a spatially non-uniform torque on the second DW whose energetically stable shape and position show therefore a non-uniform dependence on the distance from the first DW. This is different from the spin-torque transfer in bulk spin valve systems $\underline{14}, \underline{15}, \underline{16}$ or magnetic tunnel junctions $\underline{17}, \underline{18}$ insofar as in our case the DWs spatial

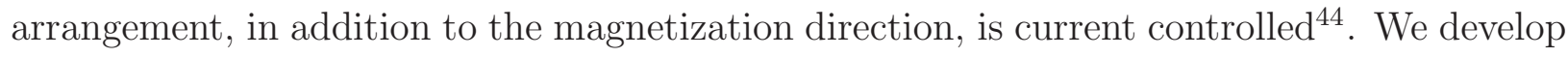
a theoretical framework to calculate the DWs current-induced effective potential and find it oscillates with the distance of DWs and their mutual polarization directions. This interaction we employ to study the DWs dynamics. As an application we propose the use of this new

effect as a tunable, current-driven two-DW magnetic nano-oscillator $19,20,21$; with a radiation emission dependent on the DWs positions in the various possible stable configurations. 


\section{THEORETICAL MODEL}

We consider a magnetic nanowire with two DWs when an electric current $I$ is transmitted through the wire (a schematic is shown in Fig. 1). When the distance $z_{0}$ between DWs is larger than the phase coherence length $L_{\phi}$, DWs act as independent scatterers. For $z_{0} \lesssim L_{\phi}$ the current transmission mediates DW coupling. For definiteness, we assume that one of the DWs (located at $z=0$ ) is pinned, e.g. by a geometric constriction, and concentrate on the effect of the current on the second DW, initially (i.e., for $I=0$ ) located at $z=z_{0}$. For $I=0$ each DW has an extension $L$. The transverse dimensions of the wire should be smaller than the exchange length and the Fermi wave length of the carriers, a situation realizable for magnetic semiconductors. The Hamiltonian $\bar{H}$ of independent carriers coupled (with a coupling constant $J$ ) to a spatially non-uniform magnetization (DWs) profile $\mathbf{M}(z)$ is modeled by (we use units with $\hbar=1$ )

$$
\bar{H}=\int d z a_{\alpha}^{\dagger}(z)\left[-\frac{\partial_{z}^{2}}{2 m} \delta_{\alpha \beta}-J \boldsymbol{\sigma}_{\alpha \beta} \cdot \mathbf{M}(z)\right] a_{\beta}(z),
$$

where $a_{\alpha}^{\dagger}$ and $a_{\alpha}$ are the creation and annihilation operators of electrons with spin $\alpha$. Applying a local gauge transformation $T(z)^{22,23,24}$ we obtain instead of the nonuniform magnetization a Zeeman splitting term and a spin-dependent spatially varying potential $U_{\alpha \beta}(z)$,

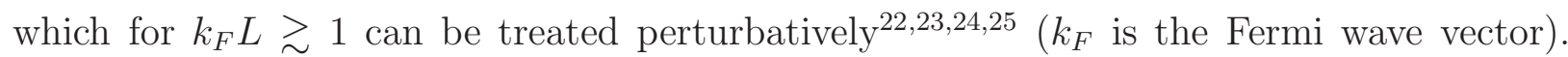
Note, for a sharp domain wall, i.e. for $k_{F} L<1$, the formalism of Ref ${ }^{26}$ can be adopted. $T(z)$ is obtained from the requirement $T^{\dagger}(z) \boldsymbol{\sigma} \cdot \mathbf{n}(z) T(z)=\sigma^{z}$, where $\mathbf{n}$ is the unit vector along $\mathbf{M}, \mathbf{M}(z)=M \mathbf{n}(z)$. The transformed Hamiltonian $H=T^{\dagger}(z) \bar{H} T(z)$ reads

$$
H=\int d z a_{\alpha}^{\dagger}(z)\left[-\frac{\partial_{z}^{2}}{2 m} \delta_{\alpha \beta}+U_{\alpha \beta}(z)-J M \sigma_{\alpha \beta}^{z}\right] a_{\beta}(z)
$$

with the perturbation given by

$$
U(z)=-\frac{1}{2 m}\left[2 A \partial_{z}+\left(\partial_{z} A\right)+A^{2}\right]
$$

and $A(z)=T^{\dagger}(z) \nabla_{z} T(z)$ is a gauge potential. For a wire with two DWs we parametrize the magnetization profile by the angles $\varphi(z)$ and $\theta(z)$ (cf. Fig 1)

$$
\begin{aligned}
& \mathbf{n}(z)=(\cos \theta \sin \varphi, \sin \theta \sin \varphi, \cos \varphi), \\
& \varphi(z)=\underbrace{\cos ^{-1}\left(\tanh \left[\frac{z}{L}\right]\right)}_{=-\varphi_{1}(z)}+\underbrace{\cos ^{-1}\left(\tanh \left[\frac{z-z_{0}}{L}\right]\right)}_{=-\varphi_{2}(z)} .
\end{aligned}
$$


(See reference ${ }^{27}$ and references therein.) The angle $\theta(z)$ describes the relative orientation between the wall pinned at $z=0$ and the other situated around $z=z_{0}$. We set $\theta_{1}$ to zero at the first wall and $\theta_{2}=\theta_{0}$ around the second (see Fig. 1 ). For $\theta_{0}=\pi$ the walls are antialigned. For $z_{0} \leq L$ DWs may merge, hence we consider the case $z_{0}>L$ for which we may write $U(z) \approx U_{1}(z)+U_{2}(z)$, where $(j=1,2)$

$$
\begin{aligned}
U_{j}(z)= & \frac{\left[\varphi_{j}^{\prime}(z)\right]^{2}}{8 m}+i \sigma^{y}\left[\frac{\varphi_{j}^{\prime \prime}(z)}{4 m}+\frac{\varphi_{j}^{\prime}(z) \partial_{z}}{2 m}\right] \cos \theta_{j} \\
& -i \sigma^{x}\left[\frac{\varphi_{j}^{\prime \prime}(z)}{4 m}+\frac{\varphi_{j}^{\prime}(z) \partial_{z}}{2 m}\right] \sin \theta_{j} .
\end{aligned}
$$

This approach is generalizable to any number of DWs, which are sufficiently far apart. As shown in Refs. ${ }^{23,24,25}$ for a single $\mathrm{DW}$, for $k_{F} L \geq 1$, i.e. when $\mathbf{M}(z)$ hardly varies within $k_{F}^{-1}$ (adiabatic DW), the terms in Eq. (6) proportional to $\varphi_{1}^{\prime \prime}(z)$ are negligibly small and a perturbative approach is appropriate for treating the electron scattering from the DWs potential (Eq. (6) $)^{45}$. Assuming $\psi^{0}(z)$ to be the wave function of an independent electron with energy $\varepsilon$ in the wire without the DWs, we find the first-order correction due to the perturbation $U_{1}(z)$, i.e. due to scattering from the first DWs, as

$$
\delta \psi_{\varepsilon}(z)=\int_{-\infty}^{\infty} d z^{\prime} G_{\varepsilon}\left(z, z^{\prime}\right) U_{1}\left(z^{\prime}\right) \psi^{0}\left(z^{\prime}\right)
$$

The Green's function $G_{\varepsilon}$ corresponds to the unperturbed Hamiltonian with $U(z)=0$. It is diagonal in spin space with elements

$$
G_{\varepsilon \sigma}\left(z, z^{\prime}\right)=-\frac{i m}{k_{\sigma}} e^{i k_{\sigma}\left|z-z^{\prime}\right|}
$$

where $k_{\sigma} \approx k_{\sigma}^{0}+\frac{i}{2 \tau_{\sigma}} \frac{m}{k_{\sigma}^{0}}$ for lifetimes $\tau_{\sigma} \gg \varepsilon_{F}^{-1}$, and $k_{\sigma}^{0}=[2 m(\varepsilon+\mu \pm J M)]^{1 / 2}$. Hence

$$
\delta \psi_{\varepsilon \uparrow}(z)=\int_{-\infty}^{\infty} d z^{\prime}\left(\begin{array}{c}
-\frac{i}{8 k_{\uparrow}} e^{i k_{\uparrow}\left|z-z^{\prime}\right|}\left[\varphi_{1}^{\prime}(z)\right]^{2} e^{i k_{\uparrow} z^{\prime}} \\
-\frac{k_{\uparrow}}{2 k_{\downarrow}} e^{i k_{\downarrow}\left|z-z^{\prime}\right|} \varphi_{1}^{\prime}(z) e^{i k_{\uparrow} z^{\prime}}
\end{array}\right)
$$

and

$$
\delta \psi_{\varepsilon \downarrow}(z)=\int_{-\infty}^{\infty} d z^{\prime}\left(\begin{array}{c}
\left.\frac{k_{\downarrow}}{2 k_{\uparrow}} e^{i k_{\uparrow}\left|z-z^{\prime}\right|} \varphi_{1}^{\prime}(z)\right] e^{i k_{\downarrow} z^{\prime}} \\
-\frac{i}{8 k_{\downarrow}} e^{i k_{\downarrow}\left|z-z^{\prime}\right|}\left[\varphi_{1}^{\prime}(z)\right]^{2} e^{i k_{\downarrow} z^{\prime}}
\end{array}\right)
$$

for incoming electrons of spin up and down, respectively.

The interaction energy of the two DWs due to the single scattered state $\psi_{\varepsilon \sigma}(z)=\psi_{\varepsilon \sigma}^{0}(z)+$ $\delta \psi_{\varepsilon \sigma}(z)$ is calculated as

$$
\Delta E_{\sigma}=\int_{-\infty}^{\infty} d z \delta \psi_{\varepsilon \sigma}^{\dagger}(z) U_{2}(z) \delta \psi_{\varepsilon \sigma}(z)
$$


Summing up the contributions of all scattering states in the energy range between $\varepsilon_{F}$ and $\varepsilon_{F}+e \Delta \phi / 2$, for an applied voltage $e \Delta \phi / 2 \ll \varepsilon_{F}$, we obtain the current-induced coupling of the DWs as

$$
\Delta E=\frac{e \Delta \phi}{\sqrt{2} \pi}\left(\frac{\Delta E_{\uparrow}}{v_{\uparrow}}+\frac{\Delta E_{\downarrow}}{v_{\downarrow}}\right)
$$

where $v_{\sigma}=k_{\sigma}^{0} / m$ is the velocity of electrons at the Fermi level.

\section{NUMERICAL EXAMPLES}

Magnetic semiconductors $\frac{7,28}{2}$ are most favorable for a sizable effect, for metallic wires the 1D limit is also within reach $\underline{29}$. Here we use in the numerical calculations similar parameters as in Ref.7, i.e. $\lambda_{F}=6 \mathrm{~nm}$; a mean free path of $l=500 \mathrm{~nm}$; an effective mass of $m=0.5 m_{e}$ ( $m_{e}$ is free electron mass); $L=\lambda_{F} ; J M=15 \mathrm{meV} ; \varepsilon_{F}=83.7 \mathrm{meV} ;$ and $e \Delta \phi=0.1 \varepsilon_{F} . \underline{46}$ The width of the wall may well be on the atomic size in the presence of constrictions $s^{5,30,31}$, i.e. well below the DW lengths in bulk materials. In such a situation, the DW-interaction increases due to the strongly enhanced DW scattering $32,33,34,35$. The interaction energy Figure 1 depends periodically on the DWs mutual angle $\theta_{0}$ and distance $z_{0}$, which results in an oscillating motion of the DW along the axis $z$ as well as an oscillating direction of DW polarization.

Now we focus on the effect of DW scattering on the electron spin density, leading to a nonequilibrium spin accumulation and to a spin torque acting on the wall. Subsequently, we study the dynamics of the DW related to the DW coupling.

The spin-density due to the single transmitted wave of spin $\sigma$ is

$$
\mathbf{S}_{\sigma}(z)=\psi_{\varepsilon \sigma}^{\dagger}(z) T(z) \boldsymbol{\sigma} T^{\dagger}(z) \psi_{\varepsilon \sigma}(z)
$$

and the total current-induced spin density is ${ }^{36}$

$$
\mathbf{S}(z)=\frac{e \phi}{2 \pi}\left(\frac{\mathbf{S}_{\uparrow}}{v_{\uparrow}}+\frac{\mathbf{S}_{\downarrow}}{v_{\downarrow}}\right) .
$$

We find that the correction to the spin density follows the magnetization profile with additional Friedel oscillations, which are a superposition of two waves with periods $k_{F \uparrow}^{-1}$ and $k_{F \downarrow}^{-1}$. The oscillations in the spin density are smaller in magnitude than the overall spin density profile and decay with increasing $z$. 

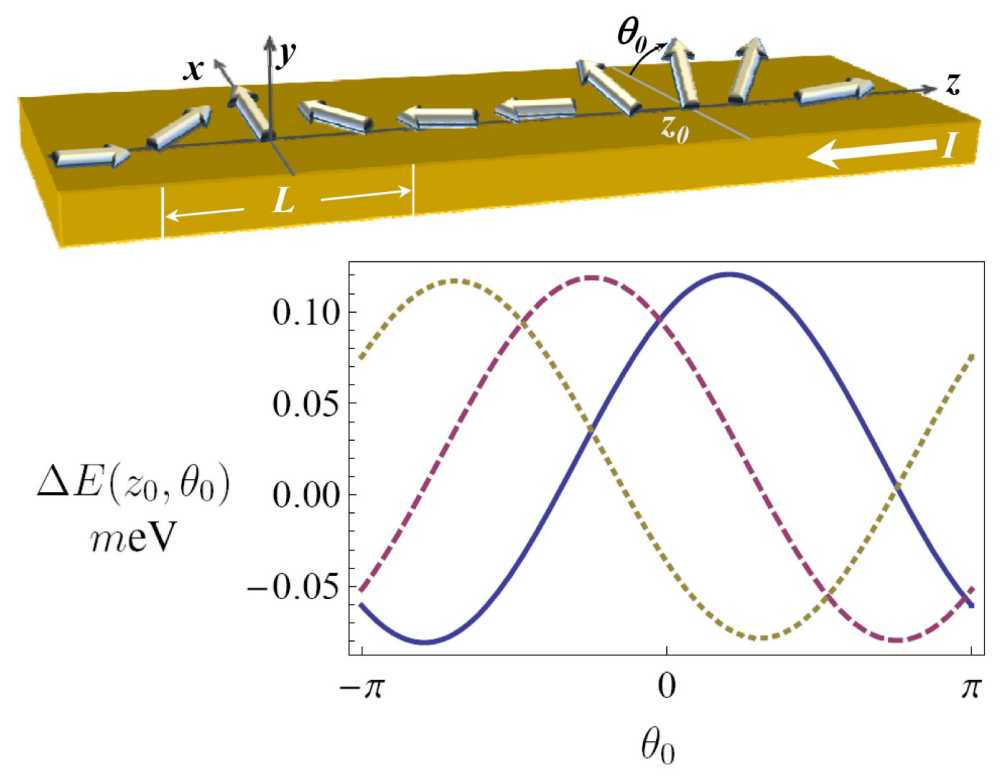

FIG. 1: (Color online) Top panel: A schematics showing the DWs magnetization profile (thick arrows). $L$ is the DW width, $z_{0}$ and $\theta_{0}$ are respectively the DW position and orientation with respect to the DW at $z=0, I$ is the current direction. Lower panel: Interaction energy $\Delta E\left(z_{0}, \theta_{0}\right)$ as a function of $z_{0}$ and $\theta_{0}$. Solid curve is for $z_{0}=30 \mathrm{~nm}$, the dashed is for $z_{0}=37.5 \mathrm{~nm}$, and the dotted is for $z_{0}=45 \mathrm{~nm}$.

We calculate the current-induced torque acting on the second DW at $z$ from

$$
\Delta \mathbf{T}\left(z, z_{0}, \theta_{0}\right)=-\frac{\gamma J}{\sigma_{c s}} \mathbf{M}\left(z, z_{0}, \theta_{0}\right) \times \Delta \mathbf{S}\left(z, z_{0}, \theta_{0}\right)
$$

where $\gamma=g \mu_{B}, g$ is the Landé factor and $\mu_{B}$ is the Bohr magneton. We assumed a thin nanowire with a cross section of $\sigma_{c s}=100 \times 20 \mathrm{~nm}^{2}$ as in Ref. $\underline{7}$. In Eq. (15) $\Delta \mathbf{S}$ is the correction to the electron spin density due to scattering. The calculated torque on the second DW is shown in Figs. 2 and 3, where $z_{0}=50 \mathrm{~L}$ and $M \approx 5.56 \times 10^{4} \mathrm{Am}^{-1}$ were used 28 . The correction to the spin torque shows that the force upon the DW depends strongly on their relative polarizations.

To inspect the current-induced dynamics of the DW at $z=z_{0}$, we evaluate the accumulated spin density that acts on the DW at $z=z_{0}$. The DW magnetization dynamics are 


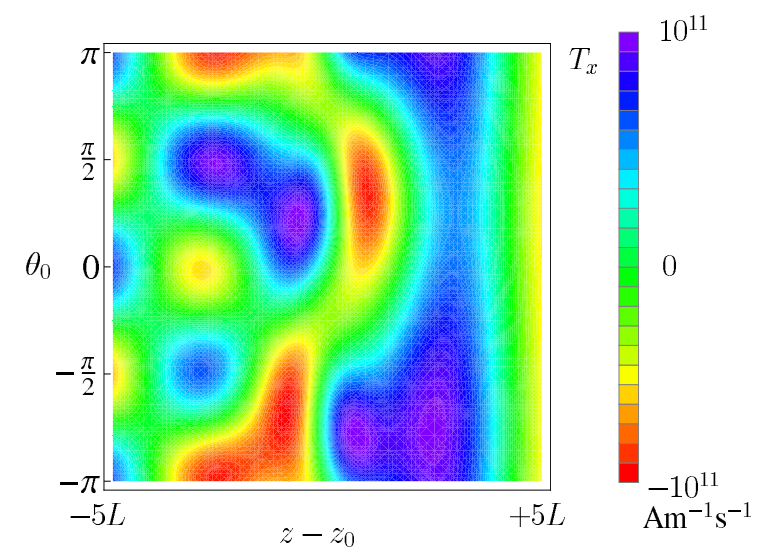

FIG. 2: (Color online) The $x$-component of the current-induced spin torque, as defined in Eq. 15, acting at the second domain wall as a function of $z$ and $\theta_{0}$.

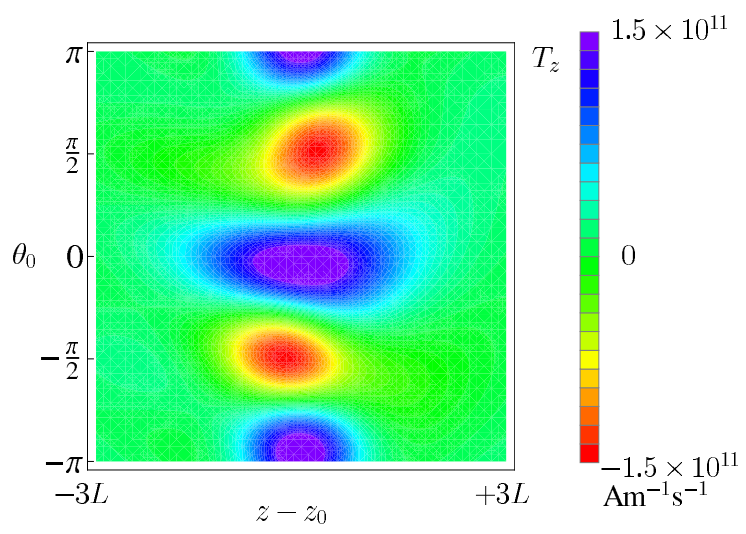

FIG. 3: (Color online) The $z$-component of the current-induced spin torque around the second domain wall as a function of $z$ and $\theta_{0}$.

then modeled using the Landau-Lifshitz equation ${ }^{47}$

$$
\partial_{t} \mathbf{M}=-\frac{\gamma J}{\sigma_{c s}} \mathbf{M} \times \mathbf{S}[\mathbf{M}]
$$

As an initial condition we assume that the magnetization profile in the wire without electric current is described by Eq. (5). The results for the time dependence of the magnetization are shown in Fig. 4 for the centre of the DW, $z=z_{0}$. We should note that the relative orientation of the walls at the start of motion does play a role in the type of motion we see. Here we present it for an arbitrary configuration. As we move away from the centre of the DW, the relative orientation becomes increasingly irrelevant. At $z=z_{0}+L$ the motion is the same regardless of the value of $\theta_{0}$.

Analyzing different magnetization components, we find their motions have different fre- 


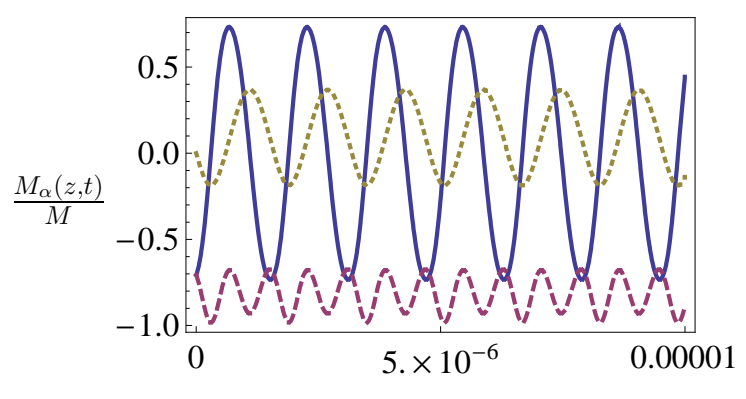

$t(\mathrm{~s})$

FIG. 4: (Color online) The time dependence of the magnetization with the initial condition for the second wall to be at an angle of $\theta_{0}=\pi / 4$ to the first wall. This is the solution to Eq. 16. The solid curve is the $x$-component, dashed the $y$-component, and dotted the $z$-component. Taken at the centre of the domain wall.

quencies and a different form. No lateral movement or permanent distortion of the DW is observed, we see only oscillations. At the edge of the DW wall there are small rotations of the magnetization, regardless of the initial conditions. If we move far from the DW then all the dynamics of the magnetization vanish.

This is in contrast to the case where we do not include the first domain wall. In this case there is no motion near the centre of the domain wall at all. Furthermore the oscillations in the magnetization towards the edge of the domain wall are much slower (by several orders of magnitude) than exhibited here.

Extending our analysis to include the effect of magnetic anisotropy we write

$$
\partial_{t} \mathbf{M}=-\frac{\gamma J}{\sigma_{c s}} \mathbf{M} \times \mathbf{S}[\mathbf{M}]+\frac{\gamma K^{\prime}}{M^{2}} \mathbf{M} \times \hat{\mathbf{x}} M_{x}
$$

We take the anisotropy constant $K^{\prime}=-10$, and therefore the $x$-axis as a hard magnetization axis. Figure 5 shows the effects of anisotropy on the domain wall motion. The anisotropy dampens motion in the $\mathrm{x}$-direction, thus exacerbating the $\mathrm{y}$ and $\mathrm{z}$ oscillations. This is also in contrast to the case where we ignore the first domain wall. In this case, although the anisotropy does introduce motion around the centre of the domain wall it does not involve a decaying $x$-component, see Fig. 6. 


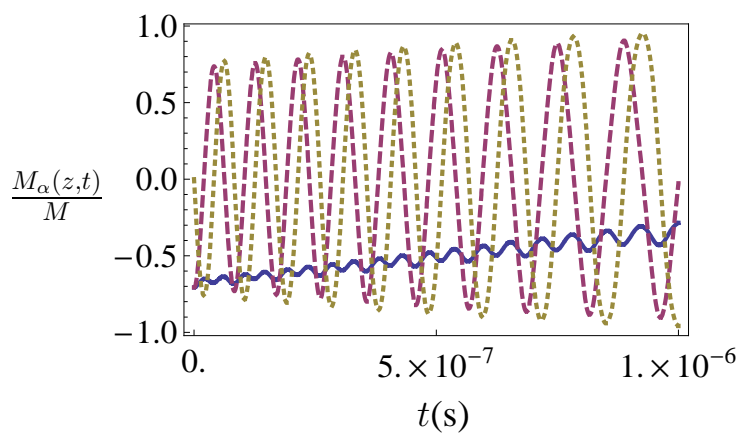

FIG. 5: (Color online) Same as in Fig. 4 when the anisotropy is included, see Eq. 17, The solid curve is the $x$-component, dashed the $y$-component, and dotted the $z$-component. Taken at the centre of the domain wall.

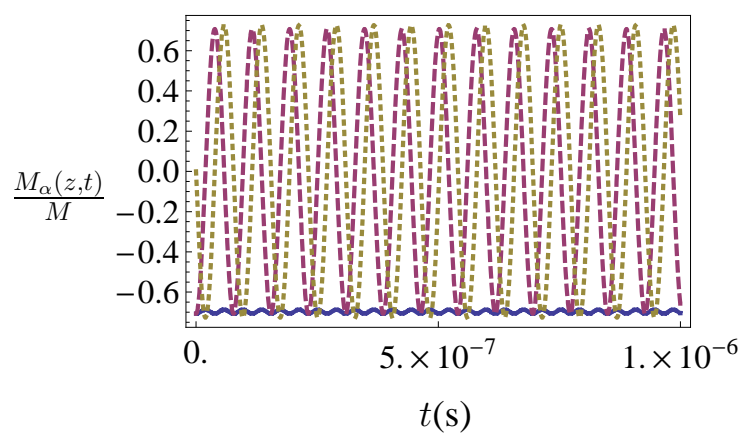

FIG. 6: (Color online) The magnetization dynamics of the second DW without the influence of the first DW, when the anisotropy is included, see Eq. 17. The solid curve is the $x$-component, dashed the $y$-component, and dotted the $z$-component. Taken at the centre of the domain wall.

\section{SUMMARY}

A current through a magnetic nanowire containing DWs results in a DW interaction mediated by the scattered charge carriers. We developed a method for calculating the interaction energy and the consequences of this new coupling mechanism. The DWs interaction energy oscillates as a function of the DWs mutual orientation and distance. This has immediate consequences on how DWs rearrange upon applying a bias voltage and on the fundamental limit of the DWs packing density. In fact, different parts of the DW oscillate at different rates and in different ways: becoming more regular, smaller, and quicker away from the DW centre. The nonequilibrium DWs oscillations around the energy minima generates radiation with a frequency dependent on the applied bias voltage, DW length and scattering strength. 
These parameters are externally tunable for utilizing the interacting DWs as a versatile

radiation source. For an experimental realization magnetic semiconductors $\frac{7}{7}, \mathbf{2}, 37,38,39,40$ are favorable, our results are in the range already achievable $e^{\underline{7}}$. Extension to the metallic case is straightforward, though DW lengths may not be easily fabricated on the required scale. In this case the results remain qualitatively similar. Furthermore anisotropy will completely dampen any DW oscillations as motion in a plane becomes much harder due to the much larger magnetization size, $M=1.72 \times 10^{6} \mathrm{Am}^{-1}$ for Fe.

In our numerical simulations we used parameters of a magnetic semiconductor with relatively large electron wavelength, $\lambda \leq L$, and much longer mean free path $l \gg \lambda$. The latter can be realized in case of small density of impurities and defects. However, magnetic semiconductors like GaMnAs are usually strongly disordered, and instead of a strong inequality one may find $l \geq \lambda$. In this case, the phase of the current-induced spin density wave at a distance $z_{0}>l$ will be affected by impurities, and, therefore, one can expect that the disorder-averaged interaction between two DWs at a distance $z_{0}$ is suppressed by the factor $e^{-z_{0} / l}$. However, we should stress that the real interaction between two DWs depends only on a given realization of the disorder and therefore is not damped by the impurities. This effect is analogous to the nondamping of the RKKY interaction between magnetic impurities in disordered metals $\underline{41} \underline{42}$. The detailed analysis of this phenomenon is beyond the scope of this paper.

\section{Acknowledgments}

This work is supported by DFG under SPP 1165, the FCT under PTDC/FIS/70843/2006 in Portugal, and by the Polish MNiSW as a research project in years $2007-2010$.

* nicholas.sedlmayr@physik.uni-halle.de

1 M. Yamanouchi, D. Chiba, F. Matsukura, and H. Ohno, Nature 428, 539 (2004).

2 A. Yamaguchi, T. Ono, S. Nasu, K. Miyake, K. Mibu, and T. Shinjo, Phys. Rev. Lett. 92, 077205 (2004).

3 S. Parkin, M. Hayashi, and L. Thomas, Science 320, 190 (2008).

4 M. Klaeui, J. Phys. Condens. Matter 20, 313001 (2008). 
5 U. Ebels, A. Radulescu, Y. Henry, L. Piraux, , and K. Ounadjela, Phys. Rev. Lett. 84, 983 (2000).

6 H. Chopra and S. Hua, Phys. Rev. B 66, 020403 (2002).

7 C. Rüster, T. Borzenko, C. Gould, G. Schmidt, L. Molenkamp, X. Liu, T. Wojtowicz, J. Furdyna, Z. Yu, and M. Flatté, Phys. Rev. Lett. 91, 216602 (2003).

8 K. Lau and W. Kohn, Surf. Sci. 75, 69 (1978).

9 A. Bogicevic, S. Ovesson, P. Hyldgaard, B. Lundqvist, H. Brune, and D. Jennison, Phys. Rev. Lett. 85, 1910 (2000).

10 K. Fichthorn and M. Scheffler, Phys. Rev. Lett. 84, 5371 (2000).

11 F. Silly, M. Pivetta, M. Ternes, F. Patthey, J. Pelz, and W. Schneider, Phys. Rev. Lett. 92, 016101 (2004).

12 N. Knorr, H. Brune, M. Epple, A. Hirstein, M. Schneider, and K. Kern, Phys. Rev. B 65, $115420(2002)$.

13 V. Stepanyuk, L. Niebergall, R. Longo, W. Hergert, and P. Bruno, Phys. Rev. B 70, 075414 (2004).

14 J. Slonczewski, J. Magn. Magn. Mater. L1, 159 (1996).

15 L. Berger, Phys. Rev. B 54, 9353 (1996).

16 J. Barnaś, A. Fert, M. Gmitra, I. Weymann, and V. Dugaev, Phys. Rev. B 72, 024426 (2005).

17 J. Slonczewski, Phys. Rev. B 39, 6995 (1989).

18 I. Theodonis, N. Kioussis, A. Kalitsov, M. Chshiev, and W. Butler, Phys. Rev. Lett. 97, 237205 (2006).

19 J. Katine, F. Albert, R. Buhrman, E. Myers, and D. Ralph, Phys. Rev. Lett. 84, 3149 (2000).

20 S. Kiselev, J. Sankey, I. Krivorotova, N. Emley, R. Schoelkopf, R. Buhrman, and D. Ralph, Nature 425, 380 (2003).

21 S. Kaka, M. Pufall, W. Rippard, T. Silva, S. Russek, and J. Katine, Nature 437, 389 (2005).

22 V. Korenman, J. Murray, and R. Prange, PRB 16, 4032 (1977).

23 G. Tatara and H. Fukuyama, PRL 78, 3773 (1997).

24 V. Dugaev, J. Barnaś, A. Łusakowski, and L. Turski, Phys. Rev. B 65, 224419 (2002).

25 V. Dugaev, V. Vieira, P. Sacramento, J. Barnaś, M. Araújo, and J. Berakdar, Int. J. Mod. Phys. 21, 1659 (2007).

26 V. Dugaev, J. Berakdar, and J. Barnaś, Phys. Rev. B 86, 104434 (2003). 
27 A. Thiavill and Y. Nakatani, Spin Dynamics in Confined Magnetic Structures III, Topics in Appl. Physics, vol. 101 (Springer-Verlag, Berlin, 2006).

28 A. Sugawara, H. Kasai, A. Tonomura, P. Brown, R. Campion, K. Edmonds, B. Gallagher, J. Zeman, and T. Jungwirth, Phys. Rev. Lett. 100, 0477202 (2008).

29 J. Schäfer, C. Blumenstein, S. Meyer, M. Wisniewski, and R. Claessen, Phys. Rev. Lett. 101, $236802(2008)$.

30 P. Bruno, Phys. Rev. Lett. 83, 2425 (1999).

31 O. Pietzsch, A. Kubetzka, M. Bode, and R. Wiesendanger, Phys. Rev. Lett. 84, 5212 (2000).

32 M. Araújo, V. Dugaev, V. Vieira, J. Berakdar, and J. Barnaś, Phys. Rev. B 74, 224429 (2006).

33 V. Dugaev, V. Vieira, P. Sacramento, J. Barnaś, M. Araújo, and J. Berakdar, Phys. Rev. B 74, 054403 (2006).

34 V. Dugaev, J. Berakdar, and J. Barnaś, Phys. Rev. Lett. 96, 047208 (2006).

35 V. Dugaev, J. Barnaś, and J. Berakdar, Phys. Rev. B 71, 024430 (2005).

36 V. Dugaev, J. Barnaś, and J. Berakdar, J. Phys. A 36, 9263 (2003).

37 H. Ohno, Science 281, 951 (1998).

38 T. Koike, , K. Hamaya, N. Funakoshi, Y. Takemura, Y. Kitamoto, and H. Munekata, IEEE Transactions on Magnetics 41, 2742 (2005).

39 T. Fukumara, H. Toyosaki, and Y. Yamada, Semiconductor Science and Technology 20, 103 (2005).

40 A. Holleitner, H. Knotz, R. Myers, A. Gossard, and D. Awschalom, Journal of Applied Physics 97, 10D314 (2005).

41 A. Y. Zyuzin and B. Z. Spivak, JETP Letters 43, 234 (1986).

42 L. N. Bulaevskii and S. V. Panyukov, JETP Letters 43, 240 (1986).

43 M. Stiles and A. Zangwill, Phys. Rev. B 66, 014407 (2002).

44 Also note, in reduced dimensions, e.g. a constrained nanowires, the propagation of the transverse component of the torque is strongly enhanced; in contrast this component is suppressed in bulk systems 43 .

45 In fact, as shown in $\frac{25}{}$, this approach is justifiable even for $k_{F} L=125$.

46 We note that the conditions for our theory to be applicable is that the mean free path $l$ should be larger than the distance $L$ between the DWs. A carrier wave length larger than $l$ means a complete localization (Ioffe-Regel criteria of localization), a case which is not of interest here. 
On the other hand, in a magnetic semiconductor, $l$ can be enhanced by lowering the acceptors density (and/or ordering them).

47 The inclusion of Gilbert damping is straightforward and has only a minor effect for the case of weak damping. 